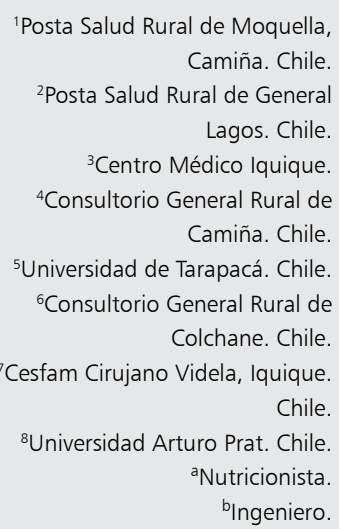

Recibido el 8 de marzo de 2016, aceptado el 12 de julio de 2016 .

Correspondencia a: Pablo Vargas Mora Consultorio General Rural de Camiña.

Arturo Prat s/n, Camiña, Región de Tarapacá. Chile.

Teléfono: (56 9) 2751772 pablovargas.med@gmail.com

\section{Factores de riesgo cardiovascular en la población Aymara rural del norte de Chile}

\author{
PABLO VARGAS M. ${ }^{1}$, STEPHANIE SAAVEDRA P. ${ }^{2}$, \\ MARÍA VIRGINIA ARAYA A. ${ }^{3}$, KHANTY LOYOLA A. ${ }^{4}$, \\ PATRICIO HUERTA G. ${ }^{5}$, MARIANA SILVA A. ${ }^{6}$, \\ SCARLETT ARAYA C. ${ }^{\text {a }}$, WILLYBALDO SAAVEDRA A. ${ }^{5}$, \\ PILAR PORTALES P. ${ }^{7}$, WILLYBALDO SAAVEDRA P. ${ }^{\mathrm{b}}$
}

\section{Prevalence of cardiovascular risk factors in a rural Aymara population from northern Chile}

Background: Some rural non-Caucasian ethnic groups have genetic protective factors for the development of chronic non-communicable diseases. Studies performed in Mapuche and Aymara ethnic groups in Chile, found significantly lower prevalence rates. Aymaras are the second most common ethnic population in Chile. Aim: To determine the prevalence of cardiovascular risk factors in a native Aymara ethnic population. Material and Methods: We studied 276 native Aymara people with a median age of 53 years (63\% women), registered in the rural clinics of Camiña and Putre. The frequency of hypertension, Type 2 Diabetes Mellitus (DM2), dyslipidemia, overweight, obesity and smoking were determined. Results: The frequency of overweight and obesity was $38 \%$ and $38.4 \%$ respectively. The prevalence of hypertension and DM2 were $18.5 \%$ and $6.9 \%$ respectively. Thirty-five percent had elevated total cholesterol, $21 \%$ had high LDL cholesterol, $48 \%$ had low HDL cholesterol and $45.7 \%$ had high triglyceride levels. Two percent smoked. Conclusions: In this group of Aymara individuals, we found a markedly lower prevalence of hypertension and DM2, despite the high prevalence of obesity and dyslipidemia.

(Rev Med Chile 2016; 144: 1144-1149)

Key words: Cardiovascular Diseases; Ethnology; Risk Factors; Rural Population.

\section{L} as enfermedades crónicas no transmisibles, representan la principal causa de morbi-mortalidad a nivel mundial, siendo las enfermedades cardiovasculares las más prevalentes ${ }^{1-3}$. En Chile, se estima que el $30 \%$ de las muertes, son provocadas por estas enfermedades, principalmente cardiopatía coronaria y accidente cerebrovascular ${ }^{4}$; ambas, patologías directamente influenciadas por factores de riesgo prevenibles y modificables, tales como: Hipertensión Arterial Crónica (HTA), Diabetes Mellitus (DM) Tipo 2, Dislipidemias, Tabaquismo, Obesidad y Sedentarismo.
Según datos entregados por la Encuesta Nacional de Salud (ENS) 2009-2010, la tasa de prevalencia de HTA en nuestro país es de $26,9 \%$, mientras que DM tipo $29,4 \%$. En el caso de las dislipidemias, la prevalencia de colesterol total (Col-total) mayor o igual a $240 \mathrm{mg} / \mathrm{dl}$ sería de $11,6 \%$, triglicéridos mayor o igual a $200 \mathrm{mg} / \mathrm{dl}$ $17 \%$, colesterol-LDL (Col-LDL) mayor o igual a $160 \mathrm{mg} / \mathrm{dl}$ 10,3\% y colesterol-HDL (Col-HDL) menor o igual a $40 \mathrm{mg} / \mathrm{dl} 28,3 \%$. Por otro lado, se estima que la obesidad tiene $25,1 \%$ de prevalencia y tabaquismo $53,4 \%{ }^{5}$. 
Hace algunos años, parecía evidente que la prevalencia de este tipo de enfermedades era mucho mayor a nivel urbano que rural, incluso doblándola ${ }^{6,7}$, lo que ratificaría el conocido efecto ambiental en el desarrollo de estas patologías, dadas por los hábitos de vida poco saludables propias de la modernización occidental. Sin embargo, esta diferencia, actualmente es controversial. En el caso de Chile, según la ENS 20092010, las cifras de HTA llegan a una prevalencia de $26,2 \%$ versus $31,7 \%$, en población urbana y rural, respectivamente, mientras que en el caso de DM tipo 2 se observa una prevalencia de $10,7 \%$ en sectores urbanos y $9,2 \%$ en rurales; en ambos casos, diferencias no significativas estadísticamente $^{5}$. Pese a esto, probablemente la "urbanización" o "aculturización" que están sufriendo las comunidades rurales en nuestro país, justifica este hallazgo ${ }^{8}$.

A nivel mundial, se han desarrollado varios estudios que indican que algunos grupos rurales étnicos no caucásicos, tendrían probablemente factores genéticos protectores, que justificarían las bajas tasas de prevalencia de enfermedades cardiovasculares, tales como grupos de China ${ }^{9}$, África Sub-Sahariana ${ }^{10}$ e India ${ }^{11}$, con prevalencias de Síndrome Metabólico de 0,9\%, 1,8\% y 18,3\%, respectivamente.

En Chile, se han realizado algunos estudios, en grupos étnicos mapuches y aymaras ${ }^{12-14}$. En estos últimos, se han encontrado tasas de prevalencia considerablemente bajas, llegando en el caso de DM tipo 2, a 1,3\% en hombres y 1,7\% en mujeres, siendo las menores de América Latina. Situación similar, se ha observado en el caso de HTA, con una prevalencia de 19,2\% en hombres y $17,7 \%$ en mujeres ${ }^{15}$.

La población aymara representa la segunda etnia más frecuente en Chile, llegando a 7\% de los pueblos indígenas y encontrándose distribuidos el 84,4\% en la región de Arica y Parinacota y región de Tarapacá, según el CENSO 2002 ${ }^{16}$. Habitan principalmente en poblados y comunidades de la precordillera y altiplano, de comunas tales como Putre, General Lagos, Camiña y Colchane.

Por esto, resulta interesante evaluar la prevalencia actual de factores de riesgo cardiovascular en esta población nativa de etnia aymara, y aportar datos importantes para una eventual re-orientación de los programas de salud pública, en relación a los pueblos originarios.

\section{Metodología}

Estudio descriptivo, de corte transversal, realizado de forma simultánea en las comunas de Camiña y Putre, entre abril y septiembre del año 2015.

Se estudió a la población aymara nativa, de 20 años y más, con un universo de 1.216 individuos, estimadas a partir de las cifras proyectadas para el año 2015, por el Instituto Nacional de Estadísticas (INE) y la proporción estimada de población aymara en cada comuna, que corresponde a $78 \%$ en Camiña y $86 \%$ en Putre ${ }^{17}$.

La muestra se seleccionó de forma aleatoria simple, entre los usuarios inscritos en los consultorios generales rurales de Camiña y Putre, incluyendo 186 y 90 pacientes, respectivamente, con un total de 276 . El poder de la muestra fue de $87,54 \%$, con un alfa de $95 \%$.

Como criterios de clasificación del origen aymara, se utilizó: al menos un apellido corroborado en registros de la Corporación Nacional de Desarrollo Indígena (CONADI) y autoidentificación con la etnia. Además, se cumple per se un tercer criterio, que es habitar un territorio reconocidamente perteneciente a la etnia.

Criterios de inclusión: población aymara de 20 años y más seleccionada, que acepta participar y firma el consentimiento informado.

Criterios de exclusión: pacientes embarazadas; residencia parcial en la comuna, definida como residencia durante más de $50 \%$ del tiempo en sectores urbanos o en alguna comuna no incluida en este estudio.

Se revisó la ficha de los pacientes seleccionados, evaluando: sexo y edad (20-44 años, 45-64 años y 65 años o más); Diagnóstico de HTA y DM tipo 2; dislipidemia (último perfil lipídico en los últimos 6 meses); último peso y talla en los últimos 6 meses, utilizando balanza mecánica con tallímetro (Seca ${ }^{\circledR}$ 700, Alemania); índice de masa corporal (IMC), a través del índice de Quetelet, calculado a partir del peso (kilogramos), divido en talla (metros) al cuadrado; El estado nutricional se clasificó como: IMC enflaquecido menor a $18,5 \mathrm{~kg} / \mathrm{m}^{2}$; normal = $18,5-24,9 \mathrm{Kg} / \mathrm{m}^{2}$; sobrepeso 25,0 a $29,9 \mathrm{Kg} / \mathrm{m}^{2}$ y obesidad mayor o igual a $30 \mathrm{Kg} / \mathrm{m}^{2}$; Tabaquismo (Sí o No, "haber fumado al menos 100 cigarrillos en la vida").

Aquellos pacientes que no tenían diagnóstico de HTA, DM tipo 2 y/o Dislipidemias, fueron 
citados y sometidos a las siguientes evaluaciones: Control Seriado de Presión Arterial (dos mediciones de presión arterial en cada brazo, en días distintos), Glicemia en ayunas, Col-total, Triglicéridos, Col-LDL y Col-HDL. Los pacientes que no tenían peso y talla reciente, en un tiempo inferior a 6 meses fueron citados para su medición.

Para el diagnóstico de HTA, se utilizó cifras igual o mayores a $140 \mathrm{mmHg}$ y/o $90 \mathrm{mmHg}$, de presión arterial sistólica (PAS) y presión arterial diastólica (PAD), respectivamente. En el caso de $\mathrm{DM}$, se utilizaron los criterios establecidos por el MINSAL: dos glicemias en ayunas iguales o superiores a $126 \mathrm{mg} / \mathrm{dl}$, o una toma igual o mayor a 200 $\mathrm{mg} / \mathrm{dl}$ mas síntomas clásicos. Para dislipidemias, se estableció el diagnóstico con: Col-total igual o mayor de $200 \mathrm{mg} / \mathrm{dl}$, Col-LDL igual o mayor a 130 $\mathrm{mg} / \mathrm{dl}$, Triglicéridos igual o mayor a $150 \mathrm{mg} / \mathrm{dl}$ y/o Col-HDL igual o menor a $40 \mathrm{mg} / \mathrm{dl}$. Para el diagnóstico de sobrepeso y obesidad, se utilizó el IMC con valores de 25 a 29,9 y $\geq 30$, respectivamente.

El análisis estadístico se realizó con el paquete estadístico SPSS versión 20. Los resultados de las variables cualitativas se expresaron en porcentajes. Los estimadores se acompañan de sus respectivos intervalos de confianza del 95\% (IC 95\%). Para la comparación de medias entre dos grupos, con variables con distribución normal, se utilizó la prueba de T de Student para datos independientes, mientras que cuando existían más de dos grupos se utilizó ANOVA. Cuando se compararon datos cuantitativos que no seguían una distribución nor- mal, se empleó la prueba de la U de Mann-Whitney cuando se comparaban dos grupos, mientras que para más de dos grupos la prueba de la $\mathrm{H}$ de Kruskal-Wallis.

\section{Resultados}

Nuestro grupo de estudio, estuvo conformado por 174 mujeres $(63,1 \%)$ y 102 hombres $(36,9 \%)$, con un promedio de edad de 53,3 + 18,8 años.

Destaca una prevalencia global de HTA de $18,48 \%$, siendo esta de $16,67 \%$ en mujeres y $21,57 \%$ en hombres $(p=0,3)$. En el caso de DM, la prevalencia global fue de $6,88 \%$, encontrando $5,75 \%$ y $8,82 \%$, en mujeres y hombres, respectivamente $(\mathrm{p}=0,33)$. Respecto a las Dislipidemias, se observó una prevalencia en mujeres de 52,3\% $y$ en hombres de $53,92 \%(p=0,79)$, dando un valor global de 52,9\%. Los promedios de lípidos fueron: Col-Total 187,6 mg/dl, Col-LDL 106,09 mg/dl, Col-HDL 48,09 mg/dl y Triglicéridos $166,33 \mathrm{mg} / \mathrm{dl}$.

El peso promedio fue de $68,9 \mathrm{~kg}$ y la talla promedio de $155 \mathrm{~cm}$. La prevalencia de sobrepeso fue de $38,04 \%$, mientras que la de obesidad de $38,41 \%$. Se obtuvo una prevalencia de tabaquismo de $2,17 \%$, siendo $3,92 \%$ en hombres y $1,15 \%$ en mujeres (Tabla 1).

En la Tabla 2, se describen la distribución prevalencias por edad, en la que destaca una mayor prevalencia de HTA en el grupo de $\geq 65$ años y

Tabla 1. Distribución prevalencias según sexo. Comunas Camiña y Putre, 2015

\begin{tabular}{|lcccc|}
\hline & $\begin{array}{c}\text { Mujeres } \\
(\mathbf{I C ~ 9 5 \% )}\end{array}$ & $\begin{array}{c}\text { Hombres } \\
\text { (IC 95\%) }\end{array}$ & $\begin{array}{c}\text { Total } \\
\text { (IC 95\%) }\end{array}$ & p-value \\
\hline Hipertensión arterial (\%) & $\begin{array}{c}\text { 16,67 } \\
(11,82-22,98)\end{array}$ & $\begin{array}{c}21,57 \\
(14,63-30,57)\end{array}$ & $\begin{array}{c}18,48 \\
(14,32-23,50)\end{array}$ & 0,312 \\
\hline Diabetes mellitus (\%) & 5,75 & 8,82 & 6,88 & 0,331 \\
\hline Dislipidemias (\%) & $(3,03-10,38)$ & $(4,52-16,11)$ & $(4,39-10,56)$ & 0,795 \\
Sobrepeso (\%) & 52,30 & 53,92 & 52,90 & 0,410 \\
& $(44,91-59,60)$ & $(44,28-63,28)$ & $(47,01-58,71)$ & 0,410 \\
\hline Obesidad (\%) & 36,21 & 41,18 & 38,04 & $(32,51-43,90)$ \\
\hline Tabaquismo (\%) & $(29,43-43,58)$ & $(32,11-50,88)$ & 38,41 & $(32,86-44,27)$ \\
\end{tabular}


mayor prevalencia de dislipidemias y obesidad entre 45 y 64 años $(p<0,05)$.

Al comparar estado nutricional con las distintas variables, existen diferencias significativas entre las medias de los grupos, comparándolo con las variables: edad, glicemia de ayuno, PAS, PAD, colesterol total, colesterol LDL y triglicéridos (Tabla 3).

Tabla 2. Distribución prevalencias según edad, Comunas Camiña y Putre, 2015

\begin{tabular}{|c|c|c|c|c|c|}
\hline & $\begin{array}{l}20 \text { a } 44 \text { años } \\
\text { (IC } 95 \%)\end{array}$ & $\begin{array}{l}45 \text { a } 64 \text { años } \\
\text { (IC } 95 \%)\end{array}$ & $\begin{array}{l}65 \text { años y mas } \\
\text { (IC 95\%) }\end{array}$ & $\begin{array}{l}\text { Total } \\
\text { (IC 95\%) }\end{array}$ & p-value \\
\hline Hipertensión arterial (\%) & $\begin{array}{c}2,15 \\
(0,12-7,97)\end{array}$ & $\begin{array}{c}13,27 \\
(7,78-21,51)\end{array}$ & $\begin{array}{c}42,35 \\
(32,39-52,96)\end{array}$ & $\begin{array}{c}18,48 \\
(14,32-23,49)\end{array}$ & 0,001 \\
\hline Diabetes mellitus (\%) & $\begin{array}{c}2,15 \\
(0,12-7,97)\end{array}$ & $\begin{array}{c}9,18 \\
(4,71-16,73)\end{array}$ & $\begin{array}{c}9,41 \\
(4,61-17,74)\end{array}$ & $\begin{array}{c}6,88 \\
(4,39-10,55)\end{array}$ & 0,080 \\
\hline Dislipidemias (\%) & $\begin{array}{c}36,56 \\
(27,47-46,71)\end{array}$ & $\begin{array}{c}72,45 \\
(62,84-80,36)\end{array}$ & $\begin{array}{c}48,24 \\
(37,92-58,70)\end{array}$ & $\begin{array}{c}52,9 \\
(47,01-58,70)\end{array}$ & 0,010 \\
\hline Sobrepeso (\%) & $\begin{array}{c}35,48 \\
(26,50-45,62)\end{array}$ & $\begin{array}{c}38,78 \\
(29,72-48,68)\end{array}$ & $\begin{array}{c}40,0 \\
(30,23-50,64)\end{array}$ & $\begin{array}{c}38,04 \\
(32,51-43,90)\end{array}$ & 0,005 \\
\hline Obesidad (\%) & $\begin{array}{c}30,11 \\
(21,69-40,10)\end{array}$ & $\begin{array}{c}48,98 \\
(39,31-58,73)\end{array}$ & $\begin{array}{c}35,29 \\
(25,95-45,91)\end{array}$ & $\begin{array}{c}38,41 \\
(32,86-44,27)\end{array}$ & 0,093 \\
\hline Tabaquismo (\%) & $\begin{array}{c}2,15 \\
(0,12-7,97)\end{array}$ & $\begin{array}{c}2,04 \\
(0,11-7,58)\end{array}$ & $\begin{array}{c}2,35 \\
(0,14-8,68)\end{array}$ & $\begin{array}{c}2,17 \\
(0,88-4,77)\end{array}$ & 0,980 \\
\hline
\end{tabular}

Tabla 3. Factores de riesgo cardiovascular según estado nutricional, Comunas Camiña y Putre, 2015

\begin{tabular}{|c|c|c|c|c|c|}
\hline & $\begin{array}{l}\text { Enflaquecido } \\
\text { (IC 95\%) }\end{array}$ & $\begin{array}{l}\text { Normal } \\
\text { (IC 95\%) }\end{array}$ & $\begin{array}{l}\text { Sobrepeso } \\
\text { (IC 95\%) }\end{array}$ & $\begin{array}{l}\text { Obesidad } \\
\text { (IC 95\%) }\end{array}$ & p-value \\
\hline Edad (años) & $\begin{array}{c}22,00 \\
(0-47,41)\end{array}$ & $\begin{array}{c}49,67 \\
(44,14-55,18)\end{array}$ & $\begin{array}{c}54,13 \\
(50,38-57,88)\end{array}$ & $\begin{array}{c}55,13 \\
(52,16-58,09)\end{array}$ & 0,027 \\
\hline DM (\%) & $\begin{array}{c}0 \\
(0-70,97)\end{array}$ & $\begin{array}{c}1,61 \\
(0-9,41)\end{array}$ & $\begin{array}{c}9,43 \\
(5,03-16,67)\end{array}$ & $\begin{array}{c}7,76 \\
(3,78-14,78)\end{array}$ & 0,250 \\
\hline $\begin{array}{l}\text { Glicemia ayuna } \\
\text { (mg/dl) }\end{array}$ & $\begin{array}{c}78,50 \\
(0-161,09)\end{array}$ & $\begin{array}{c}77,14 \\
(74,64-79,59)\end{array}$ & $\begin{array}{c}86,54 \\
(82,23-90,86)\end{array}$ & $\begin{array}{c}85,64 \\
(81,78-89,50)\end{array}$ & 0,006 \\
\hline HTA (\%) & $\begin{array}{c}0 \\
(0-70,97)\end{array}$ & $\begin{array}{c}11,3 \\
(5,28-21,81)\end{array}$ & $\begin{array}{c}16,98 \\
(10,93-25,34)\end{array}$ & $\begin{array}{c}24,27 \\
(16,96-33,43)\end{array}$ & 0,145 \\
\hline PAS (mmHg) & $\begin{array}{c}117,00 \\
(104,29-129,71)\end{array}$ & $\begin{array}{c}112,08 \\
(108,63-115,53)\end{array}$ & $\begin{array}{c}117,42 \\
(114,53-120,42)\end{array}$ & $\begin{array}{c}120,62 \\
(117,82-123,42)\end{array}$ & 0,004 \\
\hline PAD $(\mathrm{mmHg})$ & $\begin{array}{c}65,00 \\
(28,88-103,12)\end{array}$ & $\begin{array}{c}68,41 \\
(65,85-70,97)\end{array}$ & $\begin{array}{c}69,36 \\
(67,58-71,14)\end{array}$ & $\begin{array}{c}73,47 \\
(71,68-75,25)\end{array}$ & 0,001 \\
\hline $\begin{array}{l}\text { Dislipidemias } \\
(\%)\end{array}$ & $\begin{array}{c}0 \\
(0-70,97)\end{array}$ & $\begin{array}{c}27,42 \\
(17,81-39,67)\end{array}$ & $\begin{array}{c}51,88 \\
(42,47-61,16)\end{array}$ & $\begin{array}{c}71,84 \\
(62,46-79,65)\end{array}$ & 0,000 \\
\hline $\begin{array}{l}\text { Colesterol total } \\
(\mathrm{mg} / \mathrm{dl})\end{array}$ & $\begin{array}{c}136,50 \\
(92,03-180,97)\end{array}$ & $\begin{array}{c}173,46 \\
(164,38-182,53)\end{array}$ & $\begin{array}{c}184,99 \\
(177,98-191,99)\end{array}$ & $\begin{array}{c}199,47 \\
(192,16-206,78)\end{array}$ & 0,000 \\
\hline $\begin{array}{l}\text { Colesterol LDL } \\
(\mathrm{mg} / \mathrm{dl})\end{array}$ & $\begin{array}{c}65,40 \\
(0-146,72)\end{array}$ & $\begin{array}{c}96,76 \\
(89,22-104,29)\end{array}$ & $\begin{array}{c}104,66 \\
(98,58-110,74)\end{array}$ & $\begin{array}{c}113,82 \\
(107,89-119,74)\end{array}$ & 0,001 \\
\hline $\begin{array}{l}\text { Colesterol HDL } \\
(\mathrm{mg} / \mathrm{dl})\end{array}$ & $\begin{array}{c}53,50 \\
(34,44-72,56)\end{array}$ & $\begin{array}{c}50,47 \\
(46,64-54,29)\end{array}$ & $\begin{array}{c}47,40 \\
(45,49-49,31)\end{array}$ & $\begin{array}{c}47,25 \\
(44,80-49,69)\end{array}$ & 0,277 \\
\hline $\begin{array}{l}\text { Triglicéridos } \\
(\mathrm{mg} / \mathrm{dl})\end{array}$ & $\begin{array}{c}86,50 \\
(16,61-156-38)\end{array}$ & $\begin{array}{c}128,92 \\
(111,32-146,51)\end{array}$ & $\begin{array}{c}162,81 \\
(144,84-180,78)\end{array}$ & $\begin{array}{c}193,57 \\
(175,70-211,43)\end{array}$ & 0,35 \\
\hline
\end{tabular}

DM: Diabetes Mellitus tipo 2. HTA: Hipertensión Arterial Crónica. PAS: Presión arterial sistólica. PAD: Presión arterial diastólica. 


\section{Discusión}

Es destacable la baja prevalencia de HTA y DM encontrada en nuestro grupo de estudio, al compararla con las estadísticas nacionales, donde según la ENS, para HTA existe $31,7 \%$ y $26,9 \%$ en población rural y total, respectivamente; mientras que para DM, una tasa de $10,7 \%$ y $9,4 \%$ en estos mismos grupos. Este dato, es similar a lo descrito en la escasa literatura disponible sobre esta etnia, donde incluso las cifras de DM encontradas, serían menores a $2 \%{ }^{13}$.

$\mathrm{Al}$ analizar los resultados por sexo, obtuvimos una mayor prevalencia de HTA en hombres, mientras que en el caso de DM, esto se invierte. Por otro lado, si evaluamos las prevalencias de ambas patologías por edad, se mantienen valores considerablemente más bajos en todos los rangos etarios, incluso en los más jóvenes, lo que es comparable a lo encontrado por Santos y cols. ${ }^{15}$, aún cuando han pasado 15 años de dicho estudio, con todos los cambios socioculturales y demográficos propios de la "urbanización".

En lo que a dislipidemias respecta, destacan valores promedio de colesterol total similares a la población nacional $(189 \mathrm{mg} / \mathrm{dl})$, mientras que los triglicéridos, son considerablemente más altos en nuestro grupo aymara, dado que según la ENS el valor promedio en Chile es de 142,8 mg/dl.

Por otro lado, llama considerablemente la atención, las altas cifras de obesidad encontradas, superando en más de diez puntos porcentuales lo que se observa en la población nacional, puesto que según la ENS, la prevalencia de obesidad en la población rural llega a $29,1 \%$, mientras que en la población total a $25,1 \%$, lo que es superior a lo observado en estudios realizados en esta etnia, que describen no más de $33 \%$ de prevalencia de obesidad $^{12,15}$.

Esto, así como lo descrito sobre dislipidemias, se podría deber a una dieta predominantemente rica en cereales, con poca ingesta de frutas y verduras. Por lo tanto, cobra gran importancia caracterizar los hábitos nutricionales de esta población en futuros estudios, a través de encuestas de consumo y otras herramientas, para objetivar el real efecto de esto, además del factor actividad física, que a grandes rasgos dista del sedentarismo, considerando que se desempeñan gran parte del tiempo en labores de agricultura y pastoreo.

Otro aspecto muy destacable es la baja tasa de tabaquismo, no superando 3\%. Cabe señalar, que existe sólo un reporte en la literatura que describe este factor de riesgo, específico en la población aymara, el que llega a 9,5\% ${ }^{12}$. Culturalmente los grupos aborígenes altiplánicos, no consideran dentro de sus prácticas tradicionales, el consumo de tabaco u otras sustancias inhaladas o aspiradas, lo que probablemente pasa a segundo plano, considerando que residen habitualmente en poblados sobre los 2.000 metros de altura. Esto tendría un sentido opuesto, a lo que se señala respecto a la "occidentalización" que estarían sufriendo los grupos étnicos y/o rurales, en relación a los estilos de vida ${ }^{8}$.

Cabe señalar que, si bien este estudio se desarrolló sólo en pacientes usuarios de centros de salud de las comunas de Camiña y Putre, debido a la ruralidad extrema son grupos cerrados, en los que la mayoría de la población está inscrita en los centros de salud, superando en ambas comunas al 95\% de la población, por lo que este estudio es extrapolable a lo que sucede a nivel poblacional.

$\mathrm{Al}$ evaluar globalmente nuestro grupo estudio y compararlo con lo observado a nivel país, podemos inferir que su perfil es de una población con baja prevalencia de HTA, DM y tabaquismo, con similar tasa de dislipidemias, pero considerablemente más obesos. Por ende, esto obliga a plantear la posibilidad de factores de riesgo protectores de tipo genético en la etnia aymara, lo que ya ha sido demostrado en otros grupos étnicos de Asia y África ${ }^{9-11}$, siendo necesarias investigaciones específicas en este tema.

Existe una escasa cantidad de estudios en salud publicados en la etnia aymara, considerando que constituye la segunda etnia predominante en Chile. Por lo tanto, se requiere más investigación, para determinar las causas de este llamativo comportamiento de los factores de riesgo cardiovascular, lo que a su vez, justificaría una re-orientación de los programas de salud para los pueblos indígenas, que aparentemente tienen un perfil muy distinto a la población nacional.

\section{Referencias}

1. Berenson G, Srinivasan S, Baio W, Newman W, Tracy R, Wattigney W. Association between Multiple Cardiovascular Risk Factors and Atherosclerosis in Children and Young Adults. The Bogalusa Heart Study. N Enlg J Med 1998; 338 (23): 1650-6. 
2. Eckel R, Alberti K, Grundy S, Zimmet P. The metabolic syndrome. Lancet 2010; 375 (9710): 181-3.

3. Teo K, Lear S, Islam S, Mony P, Dehghan M, Li W, et al. Prevalence of a Healthy Lifestyle Among Individuals With Cardiovascular Disease in High-, Middle-and Low- Income Countries: The Prospective Urban Rural Epidemiology (PURE) Study. JAMA 2013; 309 (15): 1613-21.

4. Departamento de Estadísticas e Información de Salud (DEIS) y MINSAL, "Indicadores Básicos de Salud Chile 2013", (2013).

5. Ministerio de Salud. Encuesta Nacional de Salud ENS 2009-2010. 123. Disponible: http://www.redsalud.gov. cl/portal/docs/page/minsalcl/g_home/submenu_portada_2011/ens2010.pdf

6. Barceló A. La diabetes en las Américas. Boletín Epidemiológico OPS 2001; 22: 1-3.

7. King H, Aubert R, Herman W. Global Burden of Diabetes, 1995-2025: Prevalence, Numerical Estimates, and Projections. Diabetes Care 1998; 21 (9): 1414-31.

8. Carrasco E, Pérez F, Ángel B, Albala C, Santos J, Larenas $\mathrm{G}$, et al. Prevalencia de diabetes tipo 2 y obesidad en dos poblaciones aborígenes de Chile en ambiente urbano. Rev Med Chile 2004; 132: 1189-97.

9. Weng X, Liu Y, Ma J, Wang W, Yang G, Caballero B. An urban-rural comparison of the prevalence of the metabolic syndrome in Eastern China. Public Health Nutr 2007; 10 (2): 131-6.

10. Motala A, Mbanya J, Ramaiya K. Metabolic Syndrome in
sub-Saharan Africa. Ethn Dis 2009 Spring; 19 (2 Suppl 2): S2-8-10.

11. Ramachandran A, Snehalatha C, Satyavani K, Sivasankari F, Vijay V. Metabolic syndrome in urban Asian Indian adults-a population study using modified ATP III criteria. Diabetes Res Clin Pract 2003; 60 (3): 199 204.

12. Pérez F, Carrasco E, Santos J, Calvillán M, Albala C. Prevalencia de obesidad, hipertensión arterial y dislipidemia en grupos aborígenes rurales de Chile. Rev Med Chile 1999; 127 (10): 1169-75.

13. Pérez-Bravo F, Carrasco E, Santos J, Calvillán M, Larenas G, Albala C. Prevalence of type 2 diabetes and obesity in rural Mapuche population from Chile. Nutrition 2001; 17 (3): 236-8.

14. Ibáñez L, Sanzana R, Salas C, Navarrete C, Cartes-Velásquez R, Rainqueo A, et al. Prevalencia de síndrome metabólico en individuos de etnia Mapuche residentes en zonas rurales y urbanas de Chile. Rev Med Chile 2014; 142: 953-60.

15. Santos J, Pérez-Bravo F, Carrasco E, Calvillán M, Albala C. Low prevalence of type 2 Diaberes Despite a High Average Body Mass Index in the Aymara Natives From Chile. Nutrition 2001; 17 (4): 305-9.

16. Instituto Nacional de Estadísticas y Ministerio de Planificación Nacional. Estadísticas Sociales de los Pueblos Indígenas en Chile. Censo 2002.

17. Instituto Nacional de Estadísticas. Reportes Estadísticos y Comunales. Censo de Población y Vivienda 2002. 